\title{
Implementation of the Walrasian correspondence: the boundary problem
}

\author{
Olivier Bochet
}

Accepted: 18 June 2007 / Published online: 23 August 2007

(C) Springer-Verlag 2007

\begin{abstract}
Consider exchange economies in which preferences are continuous, convex and strongly monotonic. It is well known that the Walrasian correspondence is not Nash implementable: Maskin monotonicity (Maskin in Rev Econ Stud 66:23-38, 1999 ) is violated for Walrasian allocations on the boundary of the feasible set. We derive an impossibility result showing that the Walrasian correspondence is in fact not implementable in any of the solution concepts considered in the implementation literature. Next, imposing an additional domain restriction, we construct a sequential mechanism that doubly implements the Walrasian correspondence in subgame perfect and strong subgame perfect equilibrium. The mechanism is based on price-allocation announcements, and it fits the very description of Walrasian equilibrium. We thus take care of the boundary problem that was prominent in the Nash implementation literature.
\end{abstract}

Keywords Walrasian equilibrium · Implementability · Justified sensitivity · Double implementation · Subgame perfect equilibrium ·

Strong subgame perfect equilibrium

I warmly thank the editor of this journal and two anonymous referees whose comments helped to improve this paper. This paper is partially based on Chap. 2 of my $\mathrm{PhD}$ thesis completed at Brown University. I thank Francois Maniquet for comments and discussions on Sect. 3 of the paper. I also thank Roberto Serrano, Rajiv Vohra and William Thomson for helpful discussions and comments on this topic.

\section{O. Bochet $(\bowtie)$}

Maastricht University, Maastricht, The Netherlands

e-mail: o.bochet@algec.unimaas.nl

O. Bochet

CORE, Université Catholique de Louvain, Louvain la Neuve, Belgium 


\section{Introduction}

The question of the implementation of the Walrasian correspondence has received much attention over the past decades. While the Walrasian correspondence satisfies desirable requirements-e.g. efficiency, individual rationality-its strategic properties are much less appealing. Hurwicz (1972) shows that agents would not find it in their interest to honestly report their preferences or demand functions to the so-called auctioneer: the Walrasian correspondence is not strategy-proof. The news is not better even if agents have complete information about one another and behave according to the Nash equilibrium criterion. Hurwicz et al. (1995) show that Maskin monotonicity (Maskin 1999; first version dated 1977), a necessary condition for implementation of a social choice correspondence (henceforth, SCC) in Nash equilibrium, is violated by the Walrasian correspondence for allocations that are at the boundary of the feasible set. The Walrasian correspondence is badly behaved because it also depends on the shape of preferences outside of the feasible set. This is what generates the so-called boundary problem.

Two early papers in the literature on Nash implementation of the Walrasian correspondence, Hurwicz (1979) and Schmeidler (1980), go around the violation of Maskin monotonicity by constructing mechanisms in which allocations obtained off the equilibrium path may award unfeasible bundles with negative quantities to some agents. ${ }^{1}$ Giraud and Rochon (2001) construct an alternative mechanism that respects feasibility but rules out Walrasian allocations on the boundary. Alternatively, the literature also paid attention to the Nash implementation of the constrained Walrasian correspondence - see for instance Postlewaite and Wettstein (1989), Dutta et al. (1995), Tian (1992), Tian (2000), or Sotskov (2003). ${ }^{2}$ Consistent with the notion of Walrasian equilibrium, in each of these papers, strategy sets include announcements of allocations and prices. In a different literature, papers on non-cooperative bargaining such as Gale (1986a,b), or more recently Kunimoto and Serrano (2004) provide implementation in subgame perfect equilibrium of the Walrasian correspondence for economies with a continuum of agents. However, Walrasian allocations on the boundary of the feasible sets are also ruled out. Finally, Yildiz (2003) considers a bargaining procedure that covers only the two-player case, and uses assumptions such as uniqueness and interiority of Walrasian allocations. To the best of our knowledge, the papers on non-cooperative bargaining are the only one in this literature that use sequential mechanisms.

We know that the class of implementable SCCs considerably expands when one considers refinements of Nash equilibrium as solution concepts. For instance, in their seminal papers, Moore and Repullo (1988) (MR in the sequel) and Abreu and Sen

\footnotetext{
${ }^{1}$ In Hurwicz (1979) and Schmeidler (1980), the consumption set of agents is implicitly $\mathbb{R}^{\ell}$ instead of the usual non-negative orthant. Although a feasible bundle is always preferred by agents to one that gives negative quantities of some of the goods, the unfeasibility of some bundles off-the-equilibrium path allows to go around the violation of Maskin monotonicity on the boundary of the feasible set.

2 The constrained Walrasian correspondence is a supercorrespondence of the Walrasian correspondence. Thomson (1999) shows that it is the minimal monotonic extension of the Walrasian correspondence. For domains in which preferences are convex and Walrasian allocations are interior, the two correspondences coincide.
} 
(1990) (henceforth AS) show that Maskin monotonicity is no longer necessary for implementation in subgame perfect equilibrium. In MR, it is claimed that the Walrasian Correspondence is implementable in subgame perfect equilibrium when preferences are continuous, convex, and monotonic. In contradiction with their claim, we first show that, without further restrictions, the boundary problem generates an impossibility. Indeed, the Walrasian correspondence defined over this class of economies is in fact not implementable in any responsive game theoretic solution concept-and therefore not implementable in any of the solution concepts commonly studied in the literature. ${ }^{3}$

Next, we show that, by excluding non-differentiable preferences, the Walrasian correspondence is implementable in subgame perfect equilibrium. ${ }^{4}$ We then proceed to solve the boundary problem. Because MR and AS construct a canonical mechanism for the entire class of SCCs that are implementable in subgame perfect equilibrium, we could simply rely on their construction. ${ }^{5}$ Nevertheless, once a specific SCC of interest has been identified, this mechanism is not very appealing. It involves each agent reporting preference profiles, which are infinite-dimensional objects. Also, since this mechanism is not designed for any particular SCC, it does not capture the specific characteristics of the Walrasian correspondence.

However, for Nash implementation, Saijo (1988) and McKelvey (1989) show that the size of strategy sets in general mechanisms can be reduced. In particular, McKelvey (1989) shows that announcements of preference profiles are not necessary. While such reduction remains an open question for implementation in subgame perfect equilibrium, it is possible to follow a similar approach for specific SCCs and use tailor-made strategy sets-see for instance Serrano and Vohra (2002) who, in the context of exchange economies, provide a mechanism to implement the bargaining set in subgame perfect equilibrium.

Our position is that a mechanism is appealing if (i) in equilibrium, agents always get what they asked for, (ii) announcements of preference profiles are not part of the strategy sets of agents, and (iii) the mechanism corresponds closely to the description of the correspondence studied.

Our mechanism is simple, appealing, and it doubly implements the Walrasian correspondence in subgame perfect and strong subgame perfect equilibrium. Importantly, our construction is based on the notion of allocations, prices, and moves along price hyperplanes, which are central in the story behind the Walrasian correspondence. Recall that a pair composed of a feasible allocation and a price vector is a Walrasian equilibrium if each agent gets, at that allocation, the best bundle he can obtain in his budget set: no agent wants to "move" to obtain a different bundle on his budget hyperplane. This fundamental property is problematic for allocations that are on the boundary of the feasible set: moves along price hyperplanes can lead to bundles that are unfeasible. The mechanism we construct allows a better understanding of the strategic issues at stake when solving the boundary problem. Interestingly, in contrast to

\footnotetext{
3 See Sect. 2.2 for a formal definition of a responsive game theoretic solution concept.

4 Obviously, even with differentiability, the Walrasian correspondence is not Nash implementable as long as boundary allocations are not excluded.

5 Suppose we are interested in implementation of SCCs in subgame perfect equilibrium. Loosely speaking, we say that a mechanism is canonical if it is constructed for the implementation of entire classes of SCCs.
} 
implementation in Nash equilibrium, a change in the property of a boundary allocation when going from one preference profile to another-from Walrasian to not being Walrasian-is revealed through differences in the upper contour sets at that allocation.

The plan of the paper is as follows. In Sect. 2, we present the set-up. In Sect. 3, we prove our impossibility result. In Sect. 4, we present the mechanism and the implementation result. We provide some final comments in Sect. 5.

\section{The set-up}

\subsection{Economic environments}

There is a set $N \equiv\{1, \ldots, n\}, n \geq 2$, of agents, and a set $L \equiv\{1, \ldots, \ell\}$ of infinitely divisible goods. For each $i \in N$, let $\mathbb{R}_{+}^{\ell}$ be agent $i$ 's consumption set. For each $i \in N$, let $R_{i}$ be a complete and transitive binary relation on $\mathbb{R}_{+}^{\ell}$ indicating (weak) preferences. Let the associated strict preference and indifference relations be $P_{i}$ and $I_{i}$. For each $i \in N$, let $\mathcal{R}_{i}$ be agent $i$ 's set of possible preferences. Let $\mathcal{R}=\prod_{i} \mathcal{R}_{i}$ be the set of preference profiles. A typical preference profile is a list $R=\left(R_{i}\right)_{i \in N} \in \mathcal{R}$. For each $i \in N$, let $\omega_{i} \in \mathbb{R}_{+}^{\ell} \backslash\{0\}$ be $i$ 's individual endowment. The aggregate endowment is $\bar{\omega}=\sum \omega_{i} \gg 0 .{ }^{6}$ Each $i \in N$ is fully characterized by $\mathcal{R}_{i}$ and $\omega_{i}$. The only characteristics of agents unknown to the planner are the preferences. An economy is thus a preference profile.

We consider the following two domains of economies.

$\mathcal{R}^{C}$ (classical domain): For each $i \in N$, each $R_{i} \in \mathcal{R}_{i}^{C}$ is continuous, convex and strongly monotonic. ${ }^{7}$

$\mathcal{R}^{D}$ (differentiable domain): For each $i \in N$, each $R_{i} \in \mathcal{R}_{i}^{D}$ is convex, strongly monotonic and representable by a differentiable utility function.

A feasible allocation is a list of bundles $\left(x_{i}\right)_{i \in N} \in \mathbb{R}_{+}^{\ell n}$ such that $\sum x_{i} \leq \bar{\omega}$. Given $i \in N$, let $x_{i l} \in \mathbb{R}_{+}$be the lth coordinate of $x_{i}$.

Let $A \equiv\left\{x \in \mathbb{R}_{+}^{\ell n}: \sum x_{i} \leq \bar{\omega}\right\}$ be the set of feasible allocations.

Let $\partial A \equiv\left\{x \in A\right.$ : for some $i \in N$ and some $\left.l, m \in L, x_{i l}=\bar{\omega}_{l}, x_{i m}>0\right\}$ be the (upper) boundary of $A$. Likewise, let $A^{\circ}=A \backslash \partial A \equiv\{x \in A$ : for each $i \in N$ and $l \in L$, either $0 \leq x_{i l}<\bar{\omega}_{l}$, or $x_{i l}=\bar{\omega}_{l}$ implies that for each $\left.m \neq l, x_{i m}=0\right\}$ be the interior of $A .^{8}$

For each $i \in N$, let $A_{i}, \partial A_{i}$, and $A_{i}^{\circ}$ be the projections of $A, \partial A$ and $A^{\circ}$ onto agent $i$ 's consumption set.

For each $x \in \partial A$, let $J(x) \equiv\left\{i \in N: x_{i} \in \partial A_{i}\right\}$ be the set of agents who receive a bundle $x_{i}$ on the boundary of the feasible set.

Let $F \equiv\left\{x \in \mathbb{R}_{+}^{\ell n}: \sum x_{i}=\bar{\omega}\right\}$ be the set of balanced allocations.

\footnotetext{
6 We order vectors with the usual conventions, $\gg,>, \geq$.

7 A preference relation $R_{i}$ defined over $\mathbb{R}_{+}^{\ell}$ is convex if, for each $\left\{x_{i}, y_{i}\right\} \subset \mathbb{R}_{+}^{\ell}$ such that $x_{i} P_{i} y_{i}$, we have that for each $\lambda \in(0,1], \lambda x_{i}+(1-\lambda) y_{i} P_{i} y_{i}$.

A preference relation $R_{i}$ defined over $\mathbb{R}_{+}^{\ell}$ is strongly monotonic if, for each $\left\{x_{i}, y_{i}\right\} \subset \mathbb{R}_{+}^{\ell}, x_{i}>y_{i}$ implies that $x_{i} P_{i} y_{i}$.

8 Notice that our definition of interiority is not strictly speaking the interior of $A$ since some corners of the Edgeworth box may be included in $A^{\circ}$. We nevertheless stick to this terminology for convenience.
} 
A price vector is $p \in \mathbb{R}_{+}^{\ell}$ such that $\sum p_{l}=1$. Let $\Delta \equiv\left\{p \in \mathbb{R}_{+}^{\ell}: \sum p_{l}=1\right\}$ be the price simplex and $\Delta_{+} \equiv\left\{p \in \mathbb{R}_{++}^{\ell}: \sum p_{l}=1\right\}$ the set of strictly positive price vector.

For each $i \in N$, each $z_{i} \in A_{i}$, and each $p \in \Delta$, let $B_{i}\left(p, z_{i}\right) \equiv$ $\left\{x_{i} \in \mathbb{R}_{+}^{\ell}: p \cdot x_{i} \leq p \cdot z_{i}\right\}$ be the budget set of agent $i$ at price $p$ and bundle $z_{i}$, and $B_{i}\left(p, z_{i}\right) \cap A_{i} \equiv\left\{x_{i} \in A_{i}: p \cdot x_{i} \leq p \cdot z_{i}\right\}$ his constrained budget set at price $p$ and bundle $z_{i}$.

For each $x \in \partial A$, each $p \in \Delta_{+}$and each $i \in J(x)$, let $T_{i}(p, x) \equiv\left\{p^{\prime} \in \Delta_{+} \backslash\{p\}\right.$ : there exists $y_{i} \in A_{i} \backslash\left\{x_{i}\right\}$ such that $p^{\prime} \cdot y_{i}=p^{\prime} \cdot x_{i}$ and $\left.p \cdot y_{i}>p \cdot \omega_{i}\right\}$ be the set of prices at which $i$ can attain bundles $y_{i} \in B_{i}\left(p^{\prime}, x_{i}\right) \cap A_{i}$ that are not affordable in $B_{i}\left(p, \omega_{i}\right) \cap A_{i} \cdot 9$

For each $i \in N$, each $R_{i} \in \mathcal{R}_{i}$, and each $x_{i} \in \mathbb{R}_{+}^{\ell}$, let $U C\left(R_{i}, x_{i}\right) \equiv$ $\left\{y_{i} \in \mathbb{R}_{+}^{\ell}: y_{i} R_{i} x_{i}\right\}$ be the upper contour set of $R_{i}$ at $x_{i} ; \operatorname{LC}\left(R_{i}, x_{i}\right) \equiv$ $\left\{y_{i} \in \mathbb{R}_{+}^{\ell}: x_{i} R_{i} y_{i}\right\}$ be the lower contour set of $R_{i}$ at $x_{i} ; \operatorname{SUC}\left(R_{i}, x_{i}\right) \equiv$ $\left\{y_{i} \in \mathbb{R}_{+}^{\ell}: y_{i} P_{i} x_{i}\right\}$ be the strict upper contour set of $R_{i}$ at $x_{i}$; and $\operatorname{IC}\left(R_{i}, x_{i}\right) \equiv$ $\left\{y_{i} \in \mathbb{R}_{+}^{\ell}: x_{i} I_{i} y_{i}\right\}$ be the indifference curve of $R_{i}$ through $x_{i}$.

Given $R \in \mathcal{R}, x \in A$ is a Walrasian allocation for $R$ if there is $p \in \Delta$ such that for each $i \in N, x_{i} \in B_{i}\left(p, \omega_{i}\right)$, and for each $y_{i} \in B_{i}\left(p, \omega_{i}\right), x_{i} R_{i} y_{i}$. For each $R \in \mathcal{R}$, let $W(R)$ be the set of these allocations.

Likewise, Given $R \in \mathcal{R},(x, p) \in A \times \Delta$ is a Walrasian equilibrium for $R$ if for each $i \in N, x_{i} \in B_{i}\left(p, \omega_{i}\right)$, and for each $y_{i} \in B_{i}\left(p, \omega_{i}\right), x_{i} R_{i} y_{i} \cdot{ }^{10}$ For each $R \in \mathcal{R}$, let $W E(R)$ be the set of these allocation-price pairs.

A social choice correspondence is a mapping $f: \mathcal{R} \rightarrow A$ that associates to each preference profile a non-empty subset of feasible allocations.

The Walrasian correspondence $W: \mathcal{R} \rightarrow A$ associates to each economy $R \in \mathcal{R}$ its set of Walrasian allocations $W(R)$.

To conclude, we recall the definition of Maskin monotonicity and the necessary condition $C$ for subgame perfect implementation introduced in MR.

Maskin monotonicity: For each $\left\{R, R^{\prime}\right\} \subset \mathcal{R}$ and each $a \in f(R)$,

[For each $\left.i \in N, L C\left(R_{i}, a_{i}\right) \cap A_{i} \subseteq L C\left(R_{i}^{\prime}, a_{i}\right) \cap A_{i}\right] \Longrightarrow\left[a \in f\left(R^{\prime}\right)\right]$.

Condition C: For each $\left\{R, R^{\prime}\right\} \subset \mathcal{R}$ and each $a \in f(R) \backslash f\left(R^{\prime}\right)$, there exists a finite sequence $\left(a^{l}\right)_{l=1}^{k+1}$ of elements of $A$ such that,

a) For each $l=0, \ldots, k-1$, there exists $j \in N$ for whom,

$$
a_{j}^{l} R_{j} a_{j}^{l+1}
$$

b) There is $j \in N$ for whom $R_{j} \neq R_{j}^{\prime}$ and,

$$
a_{j}^{k} R_{j} a_{j}^{k+1} \text { and } a_{j}^{k+1} P_{j}^{\prime} a_{j}^{k}
$$

\footnotetext{
9 This definition will be explained in Sect. 4 and Fig. 3.

10 Notice that, both in $\mathcal{R}^{C}$ and $\mathcal{R}^{D}$, preferences are strongly montonic. Thus, for each Walrasian equilibrium $(x, p)$, we have $p \in \Delta_{+}$.
} 


\subsection{Game-form: definitions and notation}

Let $T$ be a game tree. Let $S$ be the set of nodes of $T, s_{0}$ the initial node, and $Z$ the set of terminal nodes. For each $i \in N$, let $M_{i}$ be the set of (pure) strategies, and for each $s \in S$, let $M_{i}^{s}$ be the set of strategies available to $i$ at node $s \in S$. Let $M \equiv \prod_{i} M_{i}$ be the set of strategy profiles. As is common in the implementation literature, we confine our attention to pure strategies. Let $g$, the outcome function, be a function that associates a feasible allocation with each path of play. An extensive mechanism $\Gamma \equiv(N, T, g)$ -or extensive game form-is a game with possibly simultaneous moves. ${ }^{11}$

Let $m \in M$. Let $g(m)$ be the allocation prescribed by the path induced by $m$, and $g_{i}(m)$ the $i$ th component of $g(m)$. Let $g(m, s)$ be the allocation corresponding to $m$ starting at node $s$. Let $\mathcal{G}$ be the set of mechanisms.

Let $\Gamma \in \mathcal{G}$. Given $R \in \mathcal{R}, \Gamma$ defines a non-cooperative game in extensive form $(\Gamma, R)$. For each $R \in \mathcal{R}$, a game-theoretic solution concept $E$ (henceforth solution concept) describes a set of predictions on how $\Gamma$ will be played, as a function of the agents' preferences. It is a mapping $E: \mathcal{G} \times \mathcal{R} \rightarrow 2^{M}$. For each $R \in \mathcal{R}$, let $E(\Gamma, R)$ and $E \mathcal{O}(\Gamma, R)$ be the set of $E$-equilibrium and $E$-equilibrium outcomes of $(\Gamma, R)$. The definition of solution concepts is indeed very broad and encompasses many concepts in which agents do not behave "strategically". In order to narrow the definition, we introduce a minimal requirement on solution concepts.

Responsiveness: $E$ is responsive with respect to $\Gamma \in \mathcal{G}$ if for each $\left\{R, R^{\prime}\right\} \subset \mathcal{R}$ and each $x \in E \mathcal{O}(\Gamma, R) \backslash E \mathcal{O}\left(\Gamma, R^{\prime}\right)$, there exist $i \in N$ and $\left\{y_{i}, z_{i}\right\} \subset A_{i}$ such that,

$$
y_{i} R_{i} z_{i} \text { and } z_{i} P_{i}^{\prime} y_{i}
$$

Observe that all the solution concepts commonly studied in the implementation literature — such as Nash equilibrium and any of its refinements - are responsive.

We define next the solution concepts that we consider in the paper. Let $R \in \mathcal{R}$ and $\Gamma \in \mathcal{G}$.

A subgame perfect equilibrium of $(\Gamma, R)$ is $m^{*} \in M$ such that for each $s \in S \backslash Z$, each $i \in N$, and each $m_{i} \in M_{i}$,

$$
g_{i}\left(m^{*}, s\right) R_{i} g_{i}\left(m_{i}, m_{-i}^{*}, s\right)
$$

Let $S P E(\Gamma, R)$ and $S P E \mathcal{O}(\Gamma, R)$ be the set of subgame perfect equilibrium and subgame perfect equilibrium outcomes of $(\Gamma, R)$.

A strong equilibrium of $(\Gamma, R)$ is $m^{*} \in M$ such that for each $H \subseteq N$, each $m^{\prime} \neq m$ with, for each $i \in N \backslash H, m_{i}=m_{i}^{\prime}$, then there is $j \in H$ for whom,

$$
g_{i}\left(m^{*}\right) R_{i} g_{i}\left(m^{\prime}\right)
$$

\footnotetext{
11 The definition we give encompasses both static and sequential mechanisms: a static mechanism can always be represented via an extensive mechanism with simultaneous moves.
} 
A strong subgame perfect equilibrium of $(\Gamma, R)$ is $m^{*} \in M$ such that for each proper subgame, $m^{*}$ is a strong equilibrium in that subgame. Let $\operatorname{SSPE}(\Gamma, R)$ and $S S P E \mathcal{O}(\Gamma, R)$ be the set of strong subgame perfect equilibrium and strong subgame perfect equilibrium outcomes of $(\Gamma, R)$.

A SCC $f$ is implemented by $\Gamma$ in $E$ if for each $R \in \mathcal{R}$, we have $E \mathcal{O}(\Gamma, R)=f(R)$.

A SCC $f$ is doubly implemented by $\Gamma$ in subgame perfect and strong subgame perfect equilibrium if for each $R \in \mathcal{R}$ we have, $\operatorname{SSPEO}(\Gamma, R)=\operatorname{SPEO}(\Gamma, R)=$ $f(R)$.

We define next the more general notion of implementability of a SCC $f$.

Implementability: There exist $\Gamma$ and a solution concept $E$, responsive with respect to $\Gamma$, such that $f$ is implemented by $\Gamma$ in $E$.

We need to introduce one last piece of notation. For each $i \in N$, let $\epsilon_{i} \in \mathbb{R}_{+}^{\ell} \backslash$ $\{0\}$ be such that $\omega_{i}-\epsilon_{i} \in \mathbb{R}_{+}^{\ell} \backslash\{0\}$. Such an $\epsilon_{i}$ exists since we assumed that for each $i \in N, \omega_{i}>0$. Let $\mathcal{P}$ be the set of one-to-one functions-permutationsfrom $N$ into itself, and let $\mathcal{P}^{n}$ be the set of permutation profiles. For each $i \in N$, let $\pi^{i} \in \mathcal{P}$ be a permutation and $\pi \equiv\left(\pi^{i}\right)_{i \in N}$. For each $\pi \in \mathcal{P}^{n}$, let $f(\pi) \equiv$ $\pi^{1}\left(\pi^{2}\left(\ldots\left(\pi^{i} \ldots\left(\pi^{n}\right)\right) \ldots\right)\right.$ be the ordered composition of the permutations in $\pi$. We call $f(\pi)$ a protocol. For each $\pi \in \mathcal{P}^{n}$, let $f_{i}(\pi)$ be the $i$ th agent in $f(\pi)$-i.e. $f_{1}(\pi)$ is the first agent, $f_{n}(\pi)$ the last agent in the protocol etc.

For each $i \in N$ and each $\pi \in \mathcal{P}^{n}$, we use the notation $\left(\pi^{\prime i}, \pi^{-i}\right)$ to indicate that $i$ uses $\pi^{\prime i}$, instead of $\pi^{i}$, while each $j \in N \backslash\{i\}$ uses $\pi^{j}$. Since we confine our attention to pure strategies, notice that, for each $i \in N$ and each $\left\{\pi, \pi^{*}\right\} \subset \mathcal{P}^{n}$, there exists $\pi^{\prime i} \neq \pi^{i}$ such that $f\left(\pi^{\prime i}, \pi^{-i}\right)=f\left(\pi^{*}\right)$. Permutations will be used as components of strategy sets and will play a role similar to integer devices found in many of the mechanisms used in the literature. However, unlike integer devices, $\mathcal{P}$ is a finite set which allows us to have finite strategy sets in the mechanism we construct in Sect. $4 .{ }^{12}$

Permutations capture here an idea of anonymity of the mechanism -equilibria should be independent of protocols - and work as a "king-maker" process because protocols will determine who will lead the game if it goes beyond Stage 1 of our mechanism. A similar device was used in Serrano and Vohra (1997). Permutations were used first as components of strategy spaces in the context of implementation, but in a different fashion, in Thomson (2005, first version dated 1995).

\section{An impossibility Result}

Given any responsive solution concept, implementability of a SCC requires the following property to be satisfied.

Justified sensitivity: For each $\left\{R, R^{\prime}\right\} \subset \mathcal{R}$ and each $a \in f(R) \backslash f\left(R^{\prime}\right)$, there exist $i \in N$ and $\left\{x_{i}, y_{i}\right\} \subset A_{i}$ such that,

$$
x_{i} R_{i} y_{i} \text { and } y_{i} P_{i}^{\prime} x_{i} .
$$

\footnotetext{
12 See Jackson (1992) for a criticism of integer games.
} 
Justified sensitivity is a basic necessary condition for a SCC to be implemented in some responsive solution concept. ${ }^{13}$ Suppose there exist $\left\{R, R^{\prime}\right\} \subset \mathcal{R}$ and $z \in A$ such that $z \in f(R) \backslash f\left(R^{\prime}\right)$. If $f$ satisfies implementability, there exist a mechanism $\Gamma$ and a solution concept $E$, responsive with respect to $\Gamma$, such that $f$ is implemented by $\Gamma$ in $E$. Precisely, there exists $m \in E(\Gamma, R)$ that gives $g(m)=z$. Assume that there does not exist $i \in N$ and $\left\{x_{i}, y_{i}\right\} \subset A_{i}$ such that $x_{i} R_{i} y_{i}$ and $y_{i} P_{i}^{\prime} x_{i}$. Since $f$ is implemented by $\Gamma$ in $E$, then $z \notin E \mathcal{O}\left(\Gamma, R^{\prime}\right)$. But this is in contradiction with $E$ being responsive. In fact, $f$ cannot be implemented by $\Gamma$ in $E$ : it is not possible to check information on allocations that are not feasible. ${ }^{14}$

When the domain of preference profiles is $\mathcal{R}^{C}$, the Walrasian correspondence violates justified sensitivity, and hence violates implementability. ${ }^{15}$ The problem we underline here also applies to public good economies and the Lindhal correspondence. Proposition 1 below can be adapted to that case.

Proposition 1 Suppose the domain is $\mathcal{R}^{C}$ and $n \geq 2$. Then the Walrasian correspondence violates implementability.

Proof Let $\ell=n=2$. Let $\mathcal{R} \subset \mathcal{R}^{C}, \mathcal{R}_{1}=\left\{R_{1}, R_{1}^{\prime}\right\}, \mathcal{R}_{2}=\left\{R_{2}\right\}, R=\left(R_{1}, R_{2}\right)$, $R^{\prime}=\left(R_{1}^{\prime}, R_{2}^{\prime}\right)$ with $R_{2}^{\prime}=R_{2}$ and $W: \mathcal{R} \rightarrow A$. Let $R_{1}, R_{1}^{\prime}$ and $R_{2}$ be respectively represented by the following functions:

$$
\begin{aligned}
& u_{1}\left(x_{1}, y_{1}\right)=\min \left\{x_{1}+y_{1} ; x_{1}+\frac{1}{3} y_{1}+\frac{8}{3}\right\}, u_{1}^{\prime}\left(x_{1}, y_{1}\right)=x_{1}+y_{1}, \text { and } \\
& u_{2}\left(x_{2}, y_{2} ; R_{2}\right)=2 x_{2}+y_{2} .
\end{aligned}
$$

The individual endowments are $\omega_{1}=\omega_{2}=(2,2)$.

Let $z^{*}=((1,4) ;(3,0))$ and $p^{*}=\left(\frac{2}{3}, \frac{1}{3}\right)$. Observe that $z^{*} \in W(R)$ with supporting price $p^{*}$. The situation is depicted in Fig. 1 .

Since $z^{*} \notin W\left(R^{\prime}\right)$, implementability requires justified sensitivity. However, there does not exist $i \in N$ and $\left\{x_{i}, y_{i}\right\} \subset A_{i}$ such that $x_{i} R_{i} y_{i}$ and $y_{i} P_{i}^{\prime} x_{i}$. It is easy to see that the proof can be extended to any $n$ and $\ell{ }^{16}$

\footnotetext{
13 The reader may have noticed that a preference change from $R$ to $R^{\prime}$ could also simply imply the existence of $i \in N$ and $\left\{x_{i}, y_{i}\right\} \subset A_{i}$ such that $x_{i} P_{i} y_{i}$ and $y_{i} I_{i}^{\prime} x_{i}$. By continuity and strong monotonicity of preferences, this implies that there exists $\left\{w_{i}, z_{i}\right\} \subset A_{i}$ such that $w_{i} R_{i} z_{i}$ and $z_{i} P_{i}^{\prime} w_{i}$. Therefore, whenever $R \neq R^{\prime}$, the structure of the model and the assumptions on preferences guarantee that, if preferences change within $A$, there always exists a "real" preference reversal in $A$.

14 Therefore, when a SCC $f$ violates justified sensitivity, the only hope to have $f$ implemented is to use some non-responsive solution concepts. For instance, let Truth be the truthtelling solution concept-i.e. agents always report truthfully-and $\Gamma$ be the direct mechanism in which $g=f$ and, for each $i \in N$, $M_{i}=\mathcal{R}_{i}$. Trivially, for each $R \in \mathcal{R}, \operatorname{Truth} \mathcal{O}(\Gamma, R)=f(R)$.

15 The violation of justified sensitivity implies that the Walrasian correspondence cannot be virtually implemented in any responsive solution concept. See Abreu and Sen (1991) for a definition of virtual Nash implementation.

16 It is clear that the domain $\mathcal{R} \subset \mathcal{R}^{C}$ of preferences does not have to be a cartesian product for the proof to go through.
} 
Fig. 1 An impossibility

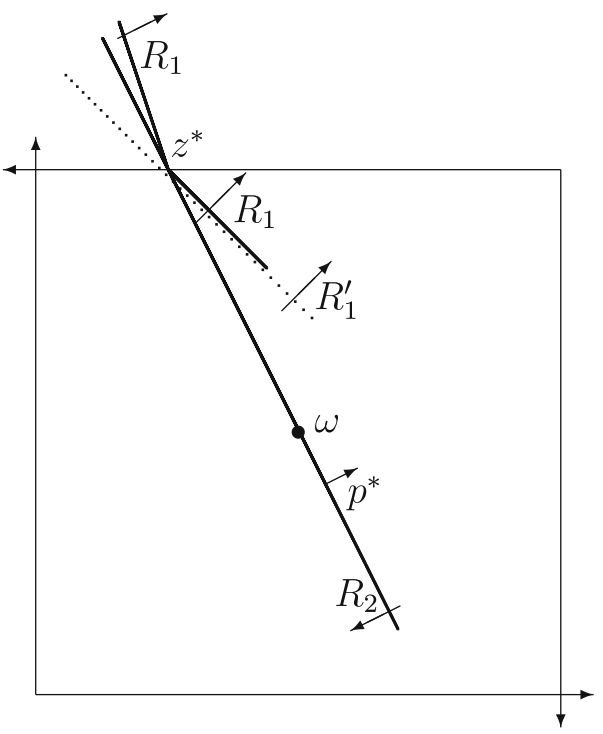

Fig. 2 Excluding kinked indifference curves

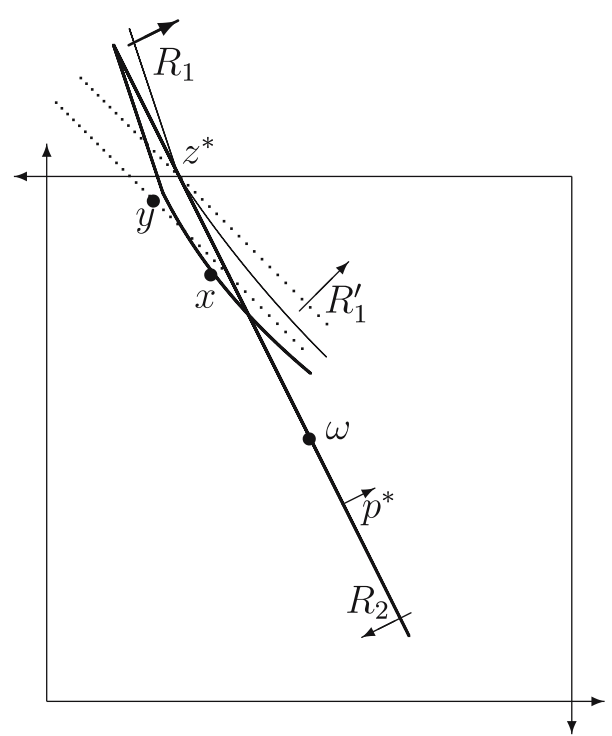

By considering the domain $\mathcal{R}^{D}$, the pathologies uncovered in Proposition 1 disappear. ${ }^{17}$ Differentiability of preferences guarantees that local information around $z^{*}$ can be used to construct a sequence $\left(a^{l}\right)_{l=1}^{k+1}$ of elements of $A$ as required by condition $C$. To see this, consider $\left\{R, R^{\prime}\right\} \subset \mathcal{R}^{D}$ and $z^{*} \in W(R) \backslash W\left(R^{\prime}\right)$ as shown in Fig. 2.

\footnotetext{
17 Obviously, even with this domain restriction, the Walrasian correspondence is not Nash implementable since corner Walrasian allocations are not excluded.
} 
We can now identify $\left\{z^{*}, x, y\right\} \subset A$ such that,

$$
z_{1}^{*} R_{1} x_{1} R_{1} y_{1} \text { and } y_{1} P_{1}^{\prime} x_{1} \text {. }
$$

When differentiability is imposed, $\operatorname{IC}\left(R_{1}, z_{1}^{*}\right) \cap A_{1}$ and $\operatorname{IC}\left(R_{1}^{\prime}, z_{1}^{*}\right) \cap A_{1}$ have to differ around $z_{1}^{*}$ if $z^{*} \notin W\left(R^{\prime}\right)$. Excluding non-differentiable preference relations from the domain, we do not need further restrictions to implement the Walrasian correspondence in subgame perfect equilibrium.

\section{Taking care of the boundary problem}

We now work with the differentiable domain $\mathcal{R}^{D}$. The mechanism we construct has three stages. At stage 1, agents simultaneously announce an allocation, a price and a permutation. First, for each $R \in \mathcal{R}^{D}$, and each $(x, p) \in A^{\circ} \times \Delta_{+}$, the information contained locally in prices is enough to determine whether $(x, p) \in W E(R)$. If $(x, p) \notin W E(R)$, there exist $i \in N$ and $y_{i} \in B_{i}\left(p, \omega_{i}\right) \cap A_{i}$ such that $y_{i} P_{i} x_{i}{ }^{18}$ The possibility of moves along budget hyperplanes is an important feature of our construction. ${ }^{19}$ Accordingly, our mechanism always stops at Stage 1 if announced allocations are in $A^{\circ}$.

However, when $x \in \partial A$, this device does not work anymore because moves along price hyperplanes can lead to infeasible bundles. To circumvent this problem, we still rely on the information contained locally in prices, but we use an alternative idea of retrading. This is where sequentiality comes into play. To see this, let us look at Fig. 3. There is $\left\{R, R^{\prime}\right\} \subset \mathcal{R}^{D}$ and $\left(z^{*}, p^{*}\right) \in \partial A \times \Delta_{+}$such that $\left(z^{*}, p^{*}\right) \in W E(R) \backslash$ $W E\left(R^{\prime}\right)$. Observe that $(i) z_{1}^{*} \in \partial A_{1}$ and (ii) $p^{\prime}$ is such that there exists $y_{1} \in A_{1}$ with $p^{\prime} \cdot y_{1}=p^{\prime} \cdot z_{1}^{*}$ and $p^{*} \cdot y_{1}>p^{*} \cdot \omega_{1}$. Hence, $1 \in J\left(z^{*}\right)$ and $p^{\prime} \in T_{1}\left(p^{*}, z^{*}\right)$. When agent 1 has preferences $R_{1}$, there exists $y_{1} \in\left(B_{1}\left(p^{\prime}, z_{1}^{*}\right) \cap A_{1}\right) \backslash B_{1}\left(p, \omega_{1}\right)$ such that $y_{1} P_{1} z_{1}^{*}$. Notice that we use a new constrained budget set $B_{1}\left(p^{\prime}, z_{1}^{*}\right) \cap A_{1}$ where $z_{1}^{*}$ plays the role of agent 1 's endowment-hence the idea of retrading. But when agent 1 has preferences $R_{1}^{\prime}$, there does not exist $y_{1} \in B_{1}\left(p^{\prime}, z_{1}^{*}\right) \cap A_{1}$ such that $y_{1} P_{1} z_{1}^{*}$. Thus $\left(z^{*}, p^{*}\right) \notin W E\left(R^{\prime}\right)$. For if $\left(z^{*}, p^{*}\right) \in W E\left(R^{\prime}\right)$, each $p^{\prime} \in T_{1}\left(p, z^{*}\right)$ would create profitable retrading opportunities for agent 1 in $B_{1}\left(p^{\prime}, z_{1}^{*}\right) \cap A_{1}$.

Whenever agents agree on an allocation $x \in \partial A$, the game continues to Stage 2 and agent $f_{1}(\pi)$ is given the opportunity to reveal whether $x$ is Walrasian for the true preference profile. The revelation is made possible because, for each $\left\{R, R^{\prime}\right\} \subset \mathcal{R}^{D}$ and each $(x, p) \in \partial A \times \Delta_{+}$such that $(x, p) \in W E(R) \backslash W E\left(R^{\prime}\right)$, there exist $i \in J(x)$ and $p^{\prime} \neq p$ such that $\operatorname{SUC}\left(R_{i}, x_{i}\right) \cap\left\{y_{i} \in A_{i}: p^{\prime} \cdot y_{i}=p^{\prime} \cdot x_{i}\right\} \neq \varnothing$ while $U C\left(R_{i}^{\prime}, x_{i}\right) \cap\left\{y_{i} \in A_{i}: p^{\prime} \cdot y_{i}=p^{\prime} \cdot x_{i}\right\}=\emptyset$. Interestingly, in contrast to implementation in Nash equilibrium, for the boundary problem, a change in the property of an allocation $x \in \partial A$ when going from one preference profile $R$ to another

\footnotetext{
18 The assumption of convexity of preferences cannot be relaxed. Thomson (1999) showed that with nonconvex preferences, the Walrasian correspondence violates Maskin monotonicity even for allocations that are in $A^{\circ}$.

19 This idea was already used, for instance, in Dutta et al. (1995) or Sotskov (2003).
} 
Fig. 3 Dealing with boundary allocations

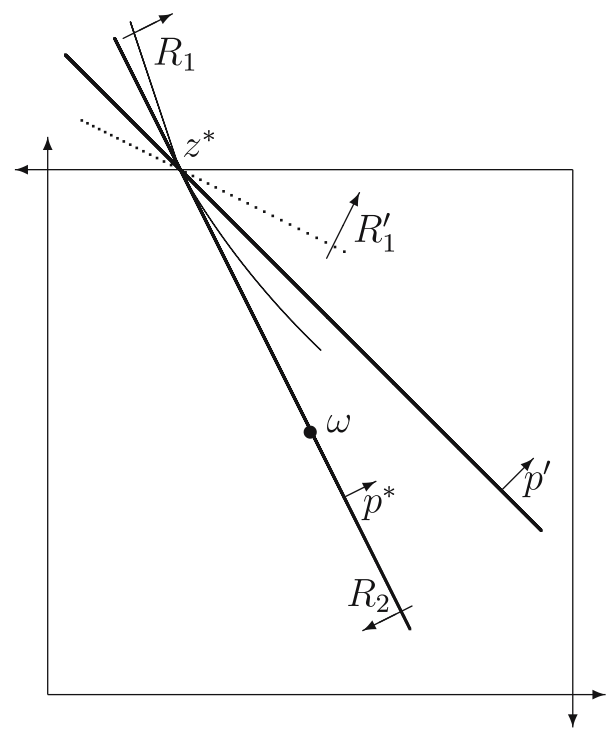

$R^{\prime}$-from being Walrasian to not being Walrasian-is revealed through differences in the upper contour sets at that allocation.

We now present formally our mechanism. It is defined for $n \geq 3$ agents. For each $i \in N$, select an $\epsilon_{i} \in \mathbb{R}_{+}^{\ell} \backslash\{0\}$ such that $\omega_{i}-\epsilon_{i} \in \mathbb{R}_{+}^{\ell} \backslash\{0\} .^{20}$

\section{Mechanism $\Gamma$ :}

Stage 1 Agents simultaneously choose $\left(m_{i}^{1}\right)_{i=1}^{n}$ with $m_{i}^{1}=\left(x^{i}, p^{i}, \pi^{i}\right) \in F \times \Delta_{+} \times \mathcal{P}$ such that, for each $i \in N$ and each $j \neq i, p^{i} \cdot x_{j}^{i}=p^{i} \cdot \omega_{j}$.

(1) If for each $i \in N,\left(x^{i}, p^{i}\right)=(\bar{x}, \bar{p})$ and $\bar{x} \in A^{\circ}$, the game stops and the outcome is $\bar{x}$.

(2) If for each $i \in N,\left(x^{i}, p^{i}\right)=(\bar{x}, \bar{p})$ and $\bar{x} \in \partial A$, then go to Stage 2 .

(3) If for each $j \in N \backslash\{i\},\left(x^{j}, p^{j}\right)=(\bar{x}, \bar{p}), i \neq f_{n}(\pi)$, and $m_{i}=\left(x^{\prime}, p^{\prime}\right) \neq$ $(\bar{x}, \bar{p})$, then (i) if $\bar{p} \cdot x_{i}^{\prime}=\bar{p} \cdot \omega_{i}$, agent $i$ gets $x_{i}^{\prime}$, each $j \in N \backslash\left\{i, f_{n}(\pi)\right\}$ gets $\frac{\bar{\omega}-x_{i}^{\prime}}{n-2}$, and $f_{n}(\pi)$ gets the 0 bundle, or (ii) if $\bar{p} \cdot x_{i}^{\prime} \neq \bar{p} \cdot \omega_{i}$, then each $j \in N$ gets his endowment $\omega_{j}$.

(4) In all other cases, the game stops, $f_{1}(\pi)$ gets $\omega_{f_{1}(\pi)}+\epsilon_{f_{n}(\pi)}$, each $j \in N \backslash$ $\left\{f_{1}(\pi), f_{n}(\pi)\right\}$ gets $\omega_{j}$, and $f_{n}(\pi)$ gets $\omega_{f_{n}(\pi)}-\epsilon_{f_{n}(\pi)}$.

Stage 2 Agent $f_{1}(\pi)$ chooses $m_{f_{1}(\pi)}^{2}=\left(k, p^{\prime}\right) \in N \backslash\left\{f_{1}(\pi)\right\} \times \Delta_{+}$.

(1) if $\bar{x}_{k} \in \partial A_{k}$ and $p^{\prime} \in T_{k}(\bar{p}, \bar{x})$, go to Stage 3 . $^{21}$

(2) In all other cases, the game stops and the outcome is $\bar{x}$.

Stage 3 Agent $k$ chooses $m_{k}^{3}=y_{k} \in\left\{y_{k}^{\prime} \in A_{k}: p^{\prime} \cdot y_{k}^{\prime}=p^{\prime} \cdot \bar{x}_{k}, \bar{p} \cdot y_{k}>\bar{p} \cdot \omega_{k}\right\} \cup\left\{\bar{x}_{k}\right\}$.

20 Individual epsilons are parameters of this mechanism.

21 That is, $p^{\prime} \in \Delta_{+}$is such that there exist feasible bundles $y_{k} \neq \bar{x}_{k}$, with $\bar{p} \cdot y_{k}=\bar{p} \cdot \omega_{k}$ and $p^{\prime} \cdot y_{k}<p^{\prime} \cdot \bar{x}_{k}$. 
(1) If he chooses $y_{k}=\bar{x}_{k}$, he gets it and agent $f_{1}(\pi)$ gets $\bar{x}_{f_{1}(\pi)}+\frac{1}{n-2}\left(\bar{\omega}-\bar{x}_{k}-\right.$ $\left.\bar{x}_{f_{1}(\pi)}\right)$. If $k \in N \backslash\left\{f_{n}(\pi)\right\}$, then each $j \in N \backslash\left\{f_{1}(\pi), k, f_{n}(\pi)\right\}$ gets $\frac{1}{n-2}(\bar{\omega}-$ $\left.\bar{x}_{k}-\bar{x}_{f_{1}(\pi)}\right)$, and $f_{n}(\pi)$ gets the 0 bundle. Otherwise, if $k=f_{n}(\pi)$, then each $j \in N \backslash\left\{f_{1}(\pi), k, f_{n-1}(\pi)\right\}$ gets $\frac{1}{n-2}\left(\bar{\omega}-\bar{x}_{k}-\bar{x}_{f_{1}(\pi)}\right)$ and agent $f_{n-1}(\pi)$ gets the 0 bundle.

(2) If he chooses $y_{k} \neq \bar{x}_{k}$, he gets it. Agent $f_{1}(\pi)$ gets the 0 bundle and each $j \in N \backslash\left\{f_{1}(\pi), k\right\}$ gets $\frac{\bar{\omega}-y_{k}}{n-2}$.

We can now proceed to the main theorem of the paper.

Theorem 1 Suppose the domain is $\mathcal{R}^{D}$ and that $n \geq 3$. Then, the Walrasian correspondence is doubly implemented by $\Gamma$ in subgame perfect and strong subgame perfect equilibrium.

Proof Let $R \in \mathcal{R}^{D}$. The proof is divided into two parts.

First part: $\operatorname{SPEO}(\Gamma, R) \subseteq W(R) .^{22}$

Let $m \in \operatorname{SPE}(\Gamma, R)$ with $m_{i}^{1}=\left(x^{i}, p^{i}, \pi^{i}\right)$. We show that $g(m) \in W(R)$. The proof is divided in several lemma.

Lemma 1 There exists $(\bar{x}, \bar{p})$ such that, for each $i \in N,\left(x^{i}, p^{i}\right)=(\bar{x}, \bar{p})$

Proof The proof is by contradiction. There are two cases.

Case 1 There exist $(\bar{x}, \bar{p}) \in A \times \Delta_{+}$and $i \in N \backslash\left\{f_{n}(\pi)\right\}$ such that for each $j \neq i$, $\left(x^{j}, p^{j}\right)=(\bar{x}, \bar{p})$, and $\left(x^{i}, p^{i}\right) \neq(\bar{x}, \bar{p})$.

First, if $\bar{p} \cdot x_{i}^{\prime}=\bar{p} \cdot \omega_{i}$, the game stops and $g_{f_{n}(\pi)}(m)=0$. Let $k \equiv f_{n}(\pi)$. Let $m_{k}^{\prime 1}=\left(x^{\prime k}, p^{\prime k}, \pi^{\prime k}\right)$ be such that $k=f_{1}\left(\pi^{\prime k}, \pi^{-k}\right)$, and $\left(x^{\prime k}, p^{\prime k}\right) \neq(\bar{x}, \bar{p})$. Then $g_{k}\left(m_{k}^{\prime}, m_{-k}\right)=\omega_{k}+\epsilon_{f_{n}\left(\pi^{\prime k}, \pi^{-k}\right)}$. Since preferences are strongly monotonic, $\omega_{k}>0$, and for each $j \in N, \epsilon_{j}>0$, this is a profitable deviation for agent $k$. Thus, $m \notin S P E(\Gamma, R)$.

Next, if $\bar{p} \cdot x_{i}^{\prime} \neq \bar{p} \cdot \omega_{i}$, the game stops and each $j \in N$ gets $g_{j}(m)=\omega_{j}$. Let $k \in N \backslash\{i\}$. Let $m_{k}^{\prime 1}=\left(x^{\prime k}, p^{\prime k}, \pi^{\prime k}\right)$ be such that $k=f_{1}\left(\pi^{\prime k}, \pi^{-k}\right)$, and $\left(x^{\prime k}, p^{\prime k}\right) \neq(\bar{x}, \bar{p})$. Then $g_{k}\left(m_{k}^{\prime}, m_{-k}\right)=\omega_{k}+\epsilon_{f_{n}\left(\pi^{\prime k}, \pi^{-k}\right)}$. Since preferences are strongly monotonic, $\omega_{k}>0$, and for each $j \in N, \epsilon_{j}>0$, this is a profitable deviation for agent $k$. Thus $m \notin S P E(\Gamma, R)$.

Case 2 Either (i) There exist $(\bar{x}, \bar{p}) \in A \times \Delta_{+}$and $k \equiv f_{n}(\pi)$ such that for each $j \in N \backslash\left\{f_{n}(\pi)\right\},\left(x^{j}, p^{j}\right)=(\bar{x}, \bar{p})$, and $\left(x^{k}, p^{k}\right) \neq(\bar{x}, \bar{p})$; or (ii) There exists $\{i, j, k\} \subset N$ such that $\left(x^{i}, p^{i}\right) \neq\left(x^{j}, p^{j}\right) \neq\left(x^{k}, p^{k}\right)$.

In either case, the game stops, each $j \in N \backslash\left\{f_{1}(\pi), f_{n}(\pi)\right\}$ gets $g_{j}(m)=\omega_{j}$, and $g_{f_{n}(\pi)}(m)=\omega_{f_{n}(\pi)}-\epsilon_{f_{n}(\pi)}$. Let $k \in N \backslash\left\{f_{1}(\pi), f_{n}(\pi)\right\}$. Let $m_{k}^{\prime 1}=\left(x^{k}, p^{k}, \pi^{\prime k}\right)$ be such that $k=f_{1}\left(\pi^{\prime k}, \pi^{-k}\right)$ and $f_{n}\left(\pi^{\prime k}, \pi^{-k}\right)=f_{n}(\pi)$. Then $g_{k}\left(m_{k}^{\prime}, m_{-k}\right)=$ $\omega_{k}+\epsilon_{f_{n}\left(\pi^{\prime k}, \pi^{-k}\right)}$. Since preferences are strongly monotonic, $\omega_{k}>0$, and for each $j \in N, \epsilon_{j}>0$, this is a profitable deviation for agent $k$. Thus, $m \notin S P E(\Gamma, R)$.

\footnotetext{
22 Since $\operatorname{SSPE}(\Gamma, R) \subseteq \operatorname{SP} E(\Gamma, R)$, it is enough, for the first part of the proof, to show that $\operatorname{SPEO}(\Gamma, R) \subseteq W E(R)$.
} 
Lemma 2 Let $\bar{x} \in A^{\circ}$. Then $(\bar{x}, \bar{p}) \in W E(R)$

Proof Suppose not. The game stops at Stage 1 with $g(m)=\bar{x} \in A^{\circ}$ but $(\bar{x}, \bar{p}) \notin$ $W E(R)$.

By definition of a Walrasian equilibrium, convexity of preferences and the fact that $\bar{x} \in A^{\circ}$, there exist $k \in N$ and $x_{k}^{\prime} \in A_{k}$ such that $\bar{p} \cdot x_{k}^{\prime}=\bar{p} \cdot \omega_{k}$ and $x_{k}^{\prime} P_{k} \bar{x}_{k}$. Let $m_{k}^{\prime 1}=\left(x^{\prime}, \bar{p}, \pi^{\prime k}\right)$ be such that $k=f_{1}\left(\pi^{\prime k}, \pi^{-k}\right)$, and for each $j \in N \backslash\{k\}, x_{j}^{\prime}=\frac{\bar{\omega}-x_{k}^{\prime}}{n-1}$. Then $g_{k}\left(m_{k}^{\prime}, m_{-k}\right)=x_{k}^{\prime}$. Since $x_{k}^{\prime} P_{k} \bar{x}_{k}$ by construction, this is a profitable deviation for agent $k$. Thus, $m \notin S P E(\Gamma, R)$.

\section{Lemma 3 Let $\bar{x} \in \partial A$. Then the game stops at Stage 2 with $\bar{x}$ as outcome}

Proof Suppose not. There exist $i \in N$ and $l, m \in L$ such that $\bar{x}_{i l}=\bar{\omega}_{l}, \bar{x}_{i m}>0$, and the game goes beyond Stage 2.

Given the rules of the game, there is $k \in\left\{f_{1}(\pi), f_{n}(\pi), f_{n-1}(\pi)\right\}$ who gets $g_{k}(m)=0$. Let $m_{k}^{\prime 1}=\left(\bar{x}, \bar{p}, \pi^{\prime k}\right)$ be such that $k=f_{1}\left(\pi^{\prime k}, \pi^{-k}\right)$ and $m_{k}^{\prime 2}=$ $\left(f_{2}\left(\pi^{\prime k}, \pi^{-k}\right), \bar{p}\right)$. The game stops with $g\left(m_{k}^{\prime}, m_{-k}\right)=\bar{x}$ as outcome.

Remember that for each $j \in N, \bar{x}_{j}>0$ since $\omega_{j}>0, \bar{p} \cdot \bar{x}_{j}=\bar{p} \cdot \omega_{j}$ and $\bar{p} \in \Delta_{+}$. Hence, by deviating, agent $k$ gets $\bar{x}_{k}>0$. By strong monotonicity of preferences, this is a profitable deviation for agent $k$. Thus, $m \notin S P E(\Gamma, R)$.

Lemma 4 Let $\bar{x} \in \partial A$. Then $(\bar{x}, \bar{p}) \in W E(R)$

Proof Suppose not. The game stops at stage 2 with $g(m)=\bar{x} \in \partial A$ but $(\bar{x}, \bar{p}) \notin$ $W E(R)$. There are two cases to consider.

Case $1 g(m)=\bar{x}$ but there exists $i \in N$ for whom,

$$
\left(B_{i}\left(\bar{p}, \omega_{i}\right) \cap A_{i}\right) \cap S U C\left(R_{i}, \bar{x}_{i}\right) \neq \emptyset .
$$

Let $k \equiv i$. Let $m_{k}^{\prime 1}=\left(x^{\prime}, \bar{p}, \pi^{\prime k}\right)$ be such that $k=f_{1}\left(\pi^{\prime k}, \pi^{k}\right), x_{k}^{\prime} \in\left(B_{k}\left(\bar{p}, \omega_{k}\right) \cap\right.$ $\left.A_{k}\right) \cap S U C\left(R_{k}, \bar{x}_{k}\right), \bar{p} \cdot x_{k}^{\prime}=\bar{p} \cdot \omega_{k}$, and for each $j \in N \backslash\{k\}, x_{j}^{\prime}=\frac{\bar{\omega}-x_{k}^{\prime}}{n-1}$. The game stops at Stage 1 and $g_{k}\left(m_{k}^{\prime}, m_{-k}\right)=x_{k}^{\prime}$. Since $x_{k}^{\prime} P_{k} \bar{x}_{k}$ by construction, this is a profitable deviation for agent $k$. Thus, $m \notin S P E(\Gamma, R)$.

Case $2 g(m)=\bar{x}$ but there exists $i \in N$ for whom,

$$
B_{i}\left(\bar{p}, \omega_{i}\right) \cap S U C\left(R_{i}, \bar{x}_{i}\right) \neq \emptyset .
$$

Since Case 1 is ruled out, we have that if $x_{i}^{\prime} \in B_{i}\left(\bar{p}, \omega_{i}\right) \cap S U C\left(R_{i}, \bar{x}_{i}\right)$, then for some $l \in L, x_{i l}^{\prime}>\bar{\omega}_{l}$. Note that $\bar{x}_{i} \in \partial A_{i}$. Let $k \in N \backslash\{i\}$. Let $m_{k}^{\prime 1}=\left(\bar{x}, \bar{p}, \pi^{\prime k}\right)$ be such that $k=f_{1}\left(\pi^{\prime k}, \pi^{k}\right)$, and $m_{k}^{\prime 2}=\left(f_{j}(\pi), p^{\prime}\right)$ be such that $i=f_{j}(\pi)$, $p^{\prime} \in T_{i}(\bar{p}, \bar{x})$, and $\left(B_{i}\left(p^{\prime}, \bar{x}_{i}\right) \cap A_{i}\right) \cap U C\left(R_{i}, \bar{x}_{i}\right)=\left\{\bar{x}_{i}\right\}$. By construction, the unique best response of $i$ at Stage 3 is $\bar{x}_{i}$. Hence, $g_{k}\left(m_{k}^{\prime}, m_{-k}\right)=\bar{x}_{k}+\frac{1}{n-2}\left(\bar{\omega}-\bar{x}_{i}-\bar{x}_{k}\right)$. Since for each $j \in N, \bar{x}_{j}>0$, and preferences are strongly monotonic, this is a profitable deviation for agent $k$. Thus, $m \notin S P E(\Gamma, R)$. 
Second part: $W(R) \subseteq S \operatorname{SPEO}(\Gamma, R)$.

Let $(x, p) \in W E(R)$. Then the following profile of strategy supports $x$ as SSPE outcome of $(\Gamma, R)$.

(i) At Stage 1 , each $i \in N$ announces $m_{i}^{1}=\left(x, p, \pi^{I}\right)$, where $\pi^{I}$ is the identity permutation.

Let $(\bar{x}, \bar{p})$ be the unanimously agreed price-allocation pair. Let $C \equiv\{i \in$ $J(\bar{x}) \backslash\left\{f_{1}(\pi)\right\}:$ (a) $\left(B_{i}\left(\bar{p}, \omega_{i}\right) \cap A_{i}\right) \cap S U C\left(R_{i}, \bar{x}_{i}\right)=\emptyset$, and (b) there is $x_{i}^{\prime} \in$ $B_{i}\left(\bar{p}, \omega_{i}\right) \cap S U C\left(R_{i}, \bar{x}_{i}\right)$ with $x_{i l}^{\prime}>\bar{\omega}_{l}$ for some $\left.l \in L\right\}$.

(ii) At Stage 2, agent $f_{1}(\pi)$ makes the following announcement:

Case 1: $C \neq \varnothing$

If $|C|=1, m_{f_{1}(\pi)}^{2}=\left(k, p^{\prime}\right) \in C \times T_{k}(\bar{p}, \bar{x})$ such that $\left(B_{k}\left(p^{\prime}, \bar{x}_{k}\right) \cap A_{k}\right) \cap$ $U C\left(R_{k}, \bar{x}_{k}\right)=\left\{\bar{x}_{k}\right\}$ and $\left(B_{k}\left(p^{\prime}, \bar{x}_{k}\right) \cap A_{k}\right) \cap S U C\left(R_{k}, \bar{x}_{k}\right)=\emptyset$.

If $|C|>1, m_{f_{1}(\pi)}^{2}=\left(k, p^{\prime}\right) \in C \times T_{k}(\bar{p}, \bar{x})$ such that for each $j \in C \backslash\{k\}$, $\bar{x}_{k} \leq \bar{x}_{j},\left(B_{k}\left(p^{\prime}, \bar{x}_{k}\right) \cap A_{k}\right) \cap U C_{k}\left(R_{k}, \bar{x}_{k}\right)=\left\{\bar{x}_{k}\right\}$, and $\left(B_{k}\left(p^{\prime}, \bar{x}_{k}\right) \cap A_{k}\right) \cap$ $S U C_{k}\left(R_{k}, \bar{x}_{k}\right)=\emptyset .^{23}$

Case 2: $C=\varnothing$

Then, $m_{f_{1}(\pi)}^{2}=\left(f_{2}(\pi), \bar{p}\right)$.

(iii) Following the choice of agent $k$ by $f_{1}(\pi)$ and the announcement of $p$, at Stage 3 , then $m_{k}^{3}=y_{k}$ such that,

$\left\{\begin{array}{l}y_{k} \neq \bar{x}_{k} \text { if there exists } y_{k} \in\left\{y_{k}^{\prime} \in A_{k}: y_{k} P_{k} \bar{x}_{k}, p^{\prime} \cdot y_{k}=p^{\prime} \cdot \bar{x}_{k}, \bar{p} \cdot y_{k}>\bar{p} \cdot \omega_{k}\right\}, \\ y_{k}=\bar{x}_{k} \text { otherwise. }\end{array}\right.$

It is clear that agent $k$ is playing a best response at Stage 3. Agent $k$ chooses the bundle he prefers between $\bar{x}_{k}$ and every possible $y_{k}$ on the budget hyperplane of $B_{k}\left(p^{\prime}, \bar{x}_{k}\right)$. In case of indifference, he favors agent $f_{1}(\pi)$ and chooses $\bar{x}_{k} \cdot{ }^{24}$ Given $(\bar{x}, \bar{p})$ agreed upon at Stage 1 and the protocol $f(\pi)$, notice that $f_{1}(\pi)$ is also playing a best response at Stage 2. He announces $p^{\prime} \neq \bar{p}$ only if there exists $k \in N \backslash\left\{f_{1}(\pi)\right\}$ for whom $\bar{x}_{f_{k}(\pi)} \in \partial A_{k},\left(B_{k}\left(\bar{p}, \omega_{k}\right) \cap A_{k}\right) \cap U C_{k}\left(R_{k}, \bar{x}_{k}\right)=\left\{\bar{x}_{k}\right\}$, and such that there is $x_{k}^{\prime} \in B_{k}\left(\bar{p}, \omega_{k}\right) \cap S U C_{k}\left(R_{k}, \bar{x}_{k}\right)$ and $l \in L$ with $x_{k l}^{\prime}>\bar{\omega}_{l}$. By doing so, agent $f_{1}(\pi)$ obtains $\bar{x}_{f_{1}(\pi)}+\frac{1}{n-2}\left(\bar{\omega}-\bar{x}_{k}-\bar{x}_{f_{1}(\pi)}\right)>\bar{x}_{f_{1}(\pi)}$. Whenever this condition is not satisfied, one of the best response of $f_{1}(\pi)$ is to announce $p^{\prime}=\bar{p}$ and to choose $f_{2}(\pi)$. Moreover, observe that a joint deviation by $f_{1}(\pi)$ and $k$ cannot make both agents better off at Stage 3 .

Finally, recall that for each Walrasian equilibrium $(x, p)$, allocation $x$ is individually rational, efficient, and such that for each $i \in N, B_{i}\left(p, \omega_{i}\right) \cap S U C_{i}\left(R_{i}, x_{i}\right)=\emptyset$. Thus, each deviation by a coalition $H \subseteq N$ results in the same outcome-for instance if agents modify the permutation they each announce—or in an outcome at which not all members of $H$ are strictly better-off than at the Walrasian allocation. Therefore, the profile of strategies described is a strong subgame perfect equilibrium of $(\Gamma, R)$.

\footnotetext{
23 Since the outcome that $f_{1}(\pi)$ gets at stage 3 can depend on the bundle that $k$ gets, the optimal choice for $f_{1}(\pi)$ is to choose $k \in C$ who is awarded the smallest bundle at $\bar{x}$.

24 This situation could happen off the equilibrium path.
} 
Hence, on the equilibrium path, each $i \in N$ announces $\left(x^{i}, p^{i}\right)=(x, p) \in$ $W E(R)$. If $x \in A^{\circ}$, the game stops at Stage 1 and $g(m)=x$. Otherwise, it goes to Stage 2 where $m_{f_{1}(\pi)}^{2}=\left(f_{2}(\pi), p\right)$. The game stops at Stage 2 and $g(m)=x$.

Remark 1 In the previous section, we underlined that the issue raised in Proposition 1 applies to the Lindhal correspondence. The mechanism we use here can be adapted to the public goods case to implement the Lindhal correspondence.

\section{Conclusion}

We have shown that, without differentiability of preferences, the Walrasian correspondence is not implementable in any of the game theoretical solution concepts commonly considered in the implementation literature. The boundary problem generates an impossibility result. Imposing differentiability of preferences, we constructed a mechanism that takes care of the boundary problem and doubly implements the Walrasian correspondence in subgame perfect and strong subgame perfect equilibrium. Our mechanism is based on price-allocation announcements and corresponds closely to the description of Walrasian equilibrium: moves along price hyperplanes are at the heart of the Walrasian equilibrium concept. The mechanism allows for a better understanding of the strategic issues attached to the boundary problem.

Finally, our mechanism can be extended to incomplete information settings by incorporating announcements of types at Stage 1 as well as state-contingent prices and allocations. An interesting issue would be to analyze the information transmission that occurs across stages and to characterize an extension of the Walrasian correspondence to incomplete information settings. Bochet (2007) follows a similar approach using a static mechanism. He finds ambiguous connections between Walrasian and Rational Expectations equilibria.

\section{References}

Abreu D, Sen A (1990) Subgame perfect implementation: a necessary and almost sufficient condition. J Econ Theory 50:285-299

Bochet O (2007) Switching from complete to incomplete information. J Math Econ 43:735-748

Dutta B, Sen A, Vohra R (1995) Nash implementation through elementary mechanisms in economic environments. Rev Econ Des 1:173-204

Gale D (1986a) Bargaining and competition. Part I: Characterization. Econometrica 54:785-806

Gale D (1986b) Bargaining and competition. Part II: Existence. Econometrica 54:807-818

Giraud G, Rochon C (2001) Consistent Collusion-Proofness and Correlation in Exchange Economies. Journal of Mathematical Economics 38:441-63

Hurwicz L (1972) On informationally decentralized system. In: McGuire CB, Radner R (eds) Decision and Organization. North-Holland, Amsterdam, pp 297-336

Hurwicz L (1979) Outcomes functions yielding Walrasian and Lindhal allocations at Nash equilibrium points. Rev Econ Stud 46:217-225

Hurwicz L, Maskin E, Postlewaite A (1995) Feasible Nash implementation of social choice rules when the designer does not know endowment or production sets. In: Ledyard JO (ed) The Economics of Informational Decentralization. Complexity, Efficiency and stability. Kluwer, Amsterdam, pp 367-433

Jackson J (1992) Implementation in undominated strategies: a look at bounded mechanisms. Rev Econ Stud 59:757-775

Kunimoto T, Serrano R (2004) Bargaining and competition revisited. J Econ Theory 115:78-88 
Maskin E (1999) Nash equilibrium and welfare optimality. Rev Econ Stud 66:23-38

McKelvey R (1989) Game forms for Nash implementation of general social choice correspondences. Soc Choice Welf 6:139-156

Moore J, Repullo R (1988) Subgame perfect implementation. Econometrica 56:1191-1120

Postlewaite A, Wettstein D (1989) Feasible and continuous implementation. Rev Econ Stud 56:603-611

Schmeidler D (1980) Walrasian analysis via strategic outcome functions. Econometrica 48:1585-1593

Serrano R, Vohra R (1997) Non-cooperative implementation of the core. Soc Choice Welf 14:513-525

Serrano R, Vohra R (2002) Bargaining and bargaining sets. Games Econ Behav 39:292-308

Saijo T (1988) Strategy space reduction in Maskin's Theorem: sufficient conditions for Nash implementation. Econometrica 56:693-700

Sotskov A (2003) Characterization of competitive allocations and the nash bargaining problem. In: Sertel M, Koray S (eds) Advances in Economic Design. Springer, Heidelberg

Thomson W (1999) Monotonic extensions on economic domains. Rev Econ Des 4:13-33

Thomson W (2005) Divide and permute and the implementation of solutions to the problem of fair division. Games Econ Behav 52:186-200

Tian G (2000) Feasible and continuous double implementation of constrained Walrasian allocations. Ann Econ Finance 1:19-32

Tian G (1992) Implementation of the Walrasian correspondence without continuous, convex, and ordered preferences. Soc Choice Welf 9:117-130

Yildiz M (2003) Walrasian bargaining. Games Econ Behav 45:465-487 